\title{
Distinctive alteration in the expression of autophagy genes in Drosophila models of amyloidopathy and tauopathy
}

\author{
Mehrnaz Haghi ${ }^{\mathrm{a}}$, Raheleh Masoudi ${ }^{\mathrm{a}}$ and Seyed Morteza Najibi ${ }^{\mathrm{b}, \mathrm{c}}$ \\ ${ }^{a}$ Department of Biology, College of Sciences, Shiraz University, Shiraz, Iran; ${ }^{b}$ Center for Molecular Protein Science, Lund University, Lund, \\ Sweden; 'Department of Statistics, College of Sciences, Shiraz University, Shiraz, Iran
}

\begin{abstract}
Background: Alzheimer's disease (AD) is one the most common types of dementia. Plaques of amyloid beta and neurofibrillary tangles of tau are two major hallmarks of AD. Metabolism of these two proteins, in part, depends on autophagy pathways. Autophagy dysfunction and protein aggregation in AD may be involved in a vicious circle. The aim of this study was to investigate whether tau or amyloid beta 42 (A 342 ) could affect expression of autophagy genes, and whether they exert their effects in the same way or not.

Methods: Expression levels of some autophagy genes, Hook, Atg6, Atg8, and Cathepsin D, were measured using quantitative PCR in transgenic Drosophila melanogaster expressing either $A \beta 42$ or Tau R406W.

Results: We found that Hook mRNA levels were downregulated in A 342 -expressing flies both 5 and 25 days old, while they were increased in 25-day-old flies expressing Tau R406W. Both Atg6 and Atg8 were upregulated at day 5 and then downregulated in 25-day-old flies expressing either A $\beta 42$ or Tau R406W. Cathepsin D expression levels were significantly increased in 5-day-old flies expressing Tau R406W, while there was no significant change in the expression levels of this gene in 5-day-old flies expressing $A \beta 42$. Expression levels of Cathepsin $D$ were significantly decreased in 25 -day-old transgenic flies expressing Tau R406W or A 342 .

Conclusion: We conclude that both $A \beta 42$ and Tau R406W may affect autophagy through dysregulation of autophagy genes. Interestingly, it seems that these pathological proteins exert their toxic effects on autophagy through different pathways and independently.
\end{abstract}

\section{ARTICLE HISTORY}

Received 6 January 2020

Revised 11 June 2020

Accepted 16 June 2020

\section{KEYWORDS}

Alzheimer's disease; amyloid beta; autophagy genes; Drosophila melanogaster; tau

\section{Introduction}

Alzheimer's disease (AD) is characterized by progressive memory impairment and dementia. This neurodegenerative disease is also one of the main causes of death in the elderly population (1). Neurofibrillary tangles (intracellular inclusion of hyperphosphorylated tau) and $A \beta$ plaques (extracellular inclusion of $A \beta 42$ peptide) are two major markers in the brain of $A D$ patients (2). The mechanism underlying $A D$ pathology is not fully understood. Some hypotheses point to these aggregations as the reason for developing $A D$ symptoms. On the other hand, recent investigations have shown that aggregate formation is a defense mechanism against soluble and aggressive forms of $A \beta$ and hyperphosphorylated tau $(3,4)$.

Although these aggregates may exert protection against their soluble oligomer forms, they block axonal/dendritic transport (5) and cause damage to mitochondrial complexes $(6,7)$, leading to increased levels of reactive oxygen species (ROS) in the brain of $A D$ patients. Autophagy (self-eating) is one of the pathways responsible for clearing these aggregations in order to prevent their accumulation (8). To eliminate the aggregates, they are transferred via a dynein/dynactin motor complex to a perinuclear aggresome. Then, they are encapsulated by neurofilaments, and the aggresome is formed. Ultimately, aggregates are cleared when the aggresome is fused to a lysosome (9). Hook protein is an adaptor facilitating the association of motor complex with its cargos (10).

Many stimuli can trigger the initiation of autophagy by ULK1/Atg1 protein kinase complex (11). This serine/threonine kinase phosphorylates Beclin1/Atg6 in the Beclin1-VPS34 PI3 kinase complex involved in nucleation of autophagosome formation (phagophore). Following Atg6 phosphorylation, VPS34 PI3 kinase can convert phosphatidylinositol (Ptdlns or $\mathrm{PI}$ ) to PI 3-phosphate (PI3P), which is essential for expansion of the autophagosome membrane $(12,13)$. Next, elongation phase and phagophore formation occur (14). Two ubiquitinlike pathways, Atg5-Atg12 and the LC3 conjugation systems, are involved in this phase. LC3 (I)/Atg8 is cleaved by Atg4 at the carboxyl-terminal site. Later, LC3 is converted to its active form, LC3II, through covalent bonding to phosphatidyl ethanolamine, which assists the fusion of the autophagosome membrane with the lysosome $(15,16)$. Ultimately, the internal 
components are degraded by lysosome hydrolases such as Cathepsin D (17-19).

Hyperphosphorylated tau and $A \beta$ aggregates can affect mitochondrial complexes and its electron chain, leading to increased levels of ROS and oxidative stress $(6,7)$. Interestingly, it has been shown that ROS is involved in the induction of autophagy in AD. Lipinski et al. reported that ROS can enhance autophagy through increase in type III PI3 kinase activity. They also confirmed that, unlike in normal aging, autophagy genes are transcriptionally increased in $A D$ patients (20). On the other hand, many studies have shown that ROS can lead to autophagy impairment by various pathways either directly or indirectly. ROS directly inhibits LC3 lipidation and also its translocation to the phagophore membrane (21). ROS increases NF- $\mathrm{KB}$ and nitric oxide (NO) synthase which, in turn, enhances NO levels (22). NO can inhibit JNK1 via S-nitrosylation at C116, leading to reduction of $\mathrm{Bcl} 2$ phosphorylation, which ultimately increases its interaction with Beclin1. This event disrupts the formation of the hVps34/Beclin1 complex, and autophagy is eventually impaired (23).

It seems that presence of protein aggregates and autophagy dysfunction in $A D$ create a vicious circle. The aim of this study was to investigate the effect of pathological Tau R406W and A 342 , two major causes of aggregate formation in AD, on the expression of autophagy genes, Atg6, Atg8, Hook, and Cathepsin D. These genes are involved in different stages of autophagy. Considering that $A D$ is an age-dependent disorder, the expression levels of autophagy genes were assessed at two different time points in A $\beta 42$ or Tau R406W transgenic Drosophila melanogaster. Tau R406W is an autosomal dominant mutation that causes tau-positive frontotemporal dementia in human. Its expression in Drosophila melanogaster, using a binary system, provides a proper tauopathy model (24).

\section{Materials and methods}

\section{Chemicals}

RNA extraction and CDNA synthesis kits were purchased from CinnaGen and Parstous companies, Iran, respectively. Brilliant II SYBR Green qPCR master mix was provided by Biofact, Germany. Other materials used in this study were provided from CinnaGen, Iran and Merck, Germany.

\section{Fly strains}

Drosophila melanogaster stocks were raised in standard rolled oats-agar medium at $22 \pm 1{ }^{\circ} \mathrm{C}, 60-70 \%$ humidity, and $12-\mathrm{h}$ light/12-h dark circadian cycle. MAPT R406W for tauopathy (expression of this protein in transgenic fly is discussed in Wittmann et al., in 2001) (24) and A $\beta 42$ for amyloidopathy (Bloomington Stock No. 33769) (25) were expressed in neurons using Dmel $\backslash$ P $\{$ GawB\}elavC155 (Bloomington Stock No. 458) driver. While expressed tau remains in the cytoplasm, presence of a signal peptide in the $A \beta 42$ construct (26) causes $A \beta 42$ transportation to the endoplasmic reticulum
(27). To investigate the pathogenesis of $A \beta 42$ and Tau R406W, GMR-Gal4 driver (Bloomington Stock No. 8605) (28) was applied to express these proteins in the fly eyes.

Flies expressing UAS-Tau R406W were from Feany's lab (Harvard Medical School, Boston, MA, USA), and all other lines were from Bloomington Drosophila Stock Centre. All crosses and their counterpart controls (parental lines) were applied in triplicate. All transgenic stocks were outcrossed to $\mathrm{w}^{1118}$ for several generations to obtain the identical genetic background of all lines, prior to the tests.

\section{Climbing assay}

For climbing assay analysis, nine groups of flies, with 10 flies per group (mix of both genders), were prepared for every single genotype. All 10 flies were transferred into a vial, and the vial was tapped gently. After each tap, flies were observed for the first $10 \mathrm{~s}$ to record the number of flies that were able to climb above the $8-\mathrm{cm}$ marked line on the vial. This assay was repeated five times for each group with a 2min interval between each measurement $(29,30)$.

\section{Drosophila eye analysis}

Transgenic (GMR-Gal4/Tau R406W and GMR-Gal4/Aß42) and control (GMR-Gal4/+) flies from at least three independent crosses ( 9 flies per group, per cross) were processed for light microscopy and image analysis with three experimental repeats.

Imaging was performed using a Nikon 80i light microscope to observe degeneration phenotype in the fly eyes. A new plugin (FLEYE) in ImageJ software was used to analyze the fly eyes. Regularity in the ommatidia was represented by probability parameter (PP) including: PP0, green; PP1, blue; PP2, yellow; PP3, orange; and finally PP4, red. Change in the colour from green to red represents the degree of reduction in eye regularity (28).

\section{Quantitative real-time PCR}

First filial generation (F1) and their parental lines (as control) were collected at 5 and 25 days after eclosion. For RNA extraction, flies were kept in acetone and stored in a $-20^{\circ} \mathrm{C}$ freezer overnight $(31,32)$. Then, flies were frozen at $-80^{\circ} \mathrm{C}$. After 10 min, flies were shaken harshly in the tube in order to separate the heads. RNA was extracted from 100 heads on liquid nitrogen by RNX plus kit following the manufacturer's protocol. RNA concentration was measured using Nano drop (Thermo Fisher Scientific), and $3 \mu \mathrm{g}$ of total RNA was reversetranscribed to CDNA. Amplification of CDNA was performed using Biofact SYBER Green master mix in an ABI 7500 PCR machine. All primers were designed as the exon-exon junction primer. RPL32 was applied as reference gene to determine the relative expression levels of Hook, Atg6, Atg8, and Cathepsin D using a 2(-delta delta C(T)) method (33). Each sample was run in triplicate. The sequences of primers are given in Supplementary Table S1. 


\section{Statistical analysis}

Here, we applied R (version 3.6.1) and SPSS (version 19) programmes to perform statistical analysis.

The number of samples in biological studies is usually small, and the assumptions of parametric statistical models cannot be achieved. One solution is to use alternative non-parametric statistical models, which have lower power compared to the parametric versions. Another solution is to use Bayesian inference, which is more powerful to infer reliable and reproducible results (34-36). In this study, because $A \beta 42$ and Tau R406W have two subgroups (cross and control), we used the Bayesian hierarchical mixture model for testing. Our hypotheses were that there are differential gene expression and climbing ability in two biologic conditions ( $A D$ and control) at different time points ( 5 and 25 days). The proposed model exploits available position-specific read counts, minimizing required data preprocessing and making maximum use of available information. Our analysis has been done by Stan language that is using a state-of-the-art algorithm known as Hamiltonian Monte Carlo (HMC), which builds upon the Metropolis-Hastings algorithm by incorporating many theoretical ideas from physics. Specifically, we used the rstanarm package in $R$ software, which is a powerful package for Bayesian hierarchical models by stan_Ime4 for estimating the model parameters. In this paper, we considered a full multilevel model for group/subgroup*time*

(genes) that means we assume random intercept and slope for each category. We had to have an estimation of different intercepts and slopes for each experimental condition, which was clearly needed based on the raw data (see Figures 1 and 2). The number of samples is selected to be 4000 (2000 for warmup), and four independent chains ran for the sake of convergency and posterior sampling evaluation. The median estimation and the highest posterior density (HPD) intervals with probability of 0.95 are reported based on 2000 samples from posterior distribution of model parameters. The Bayesian contrast estimations are evaluated by the emmeans package in $\mathrm{R}$.

(A) Raw Data of Percent of Flies above Target and Hierarchical Bayesian Fitted Models
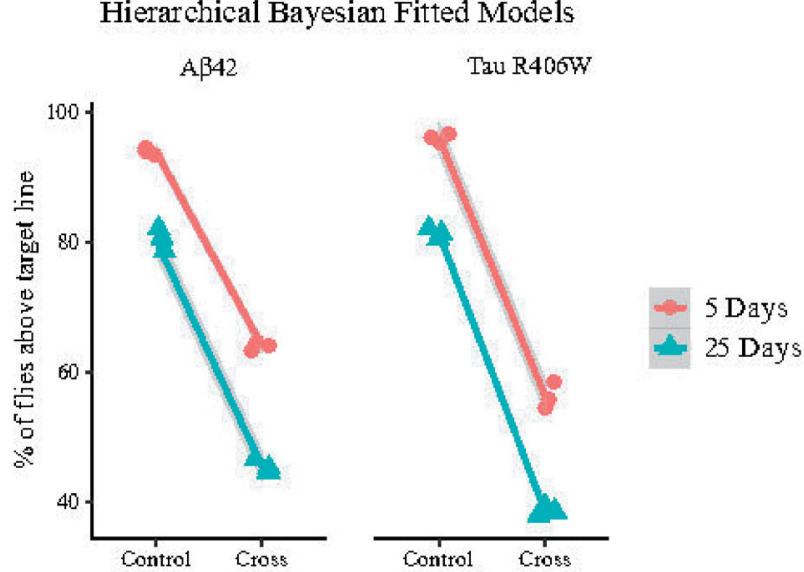

Besides the Bayesian model, we have also presented our data as fold changes when comparing crosses and their counterpart controls. Independent samples $t$ test was employed to compare the mean values. $p$ values less than 0.05 were considered as statistically significant.

\section{Results}

\section{Decrease in the climbing ability of $A \beta 42$ or Tau R406W transgenic flies}

Locomotive defects can be examined in transgenic flies as one of the AD symptoms. Here, we tested negative geotaxis ability as a behavioural assay to show that the expression of our target genes can affect the natural tendency of flies to move against gravity $(29,30)$. To assess the climbing ability, 5- and 25-day-old transgenic flies were examined. It was found that the ability of climbing in both transgenic flies was remarkably decreased compared to their controls. The raw data and Bayesian estimation of difference between controls and transgenic flies are depicted in Figure 1 (for more details see Table 1; and also Supplementary Table S2).

There was 0.32 - and 0.42 -fold decrease ( $p$ values $\leq 0.001$ ) in 5-day-old $A \beta 42$ or Tau R406W-expressing flies, respectively; 25-day-old flies expressing $A \beta 42$ or Tau R406W showed 0.44 - and 0.53 -fold reduction ( $p$ values $\leq 0.001$ ) in the climbing ability, respectively. For more details see Supplementary Figure S1 and Supplementary Table S3.

\section{Eye degeneration was observed in flies expressing either Tau R406W or A $\beta 42$}

To screen the pathogenesis of our genes of interest ( $A \beta 42$ and Tau R406W), we investigated the degeneration of Drosophila retina as a model system by expressing the transgenes using the GMR-Gal4 driver. As can be seen in Figure 3, there was more irregularity (yellow and red colors) in the eye ommatidia of transgenic flies compared to parental lines

(B) Baeysian Estimation of Control and Cross Contrast

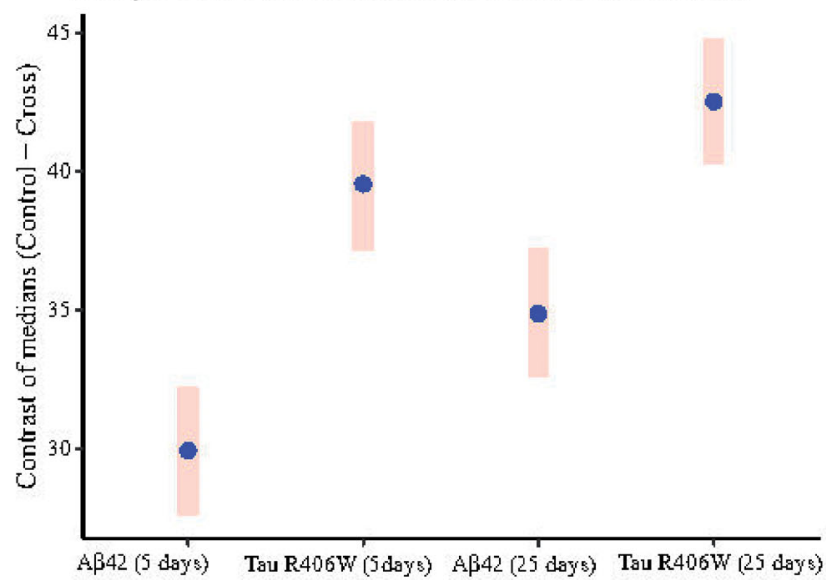

Figure 1. (A) Representing the raw data of the percent of flies (control and cross) above the target line in three independent biological repeats for 5- and 25-dayold flies and the fitted Bayesian hierarchical model lines for each group and subgroup and their shaded $95 \%$ highest posterior density (HPD) region based on 2000 posterior samplings of model parameters. (B) Bayesian estimation of contrast between medians (control-cross) and their $95 \%$ HPD region based on 2000 posterior samplings of each contrast. Because zero is not included in any of the reported HPD's intervals, there is significant difference between the control and cross negative geotaxis ability with $5 \%$ error type $(\alpha=0.05)$. 
Raw Data and Hierarchical Bayesian Fitted Models for Realative Gene Expression Fold Change

A $\beta 42$

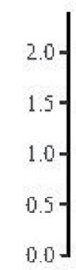

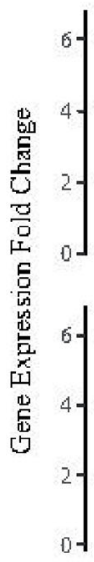

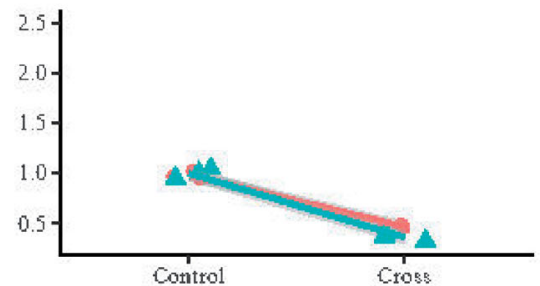

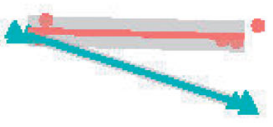

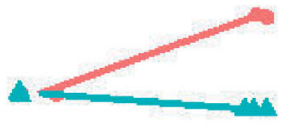

Control

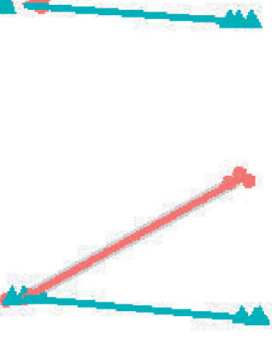

Cross
Tau R406w
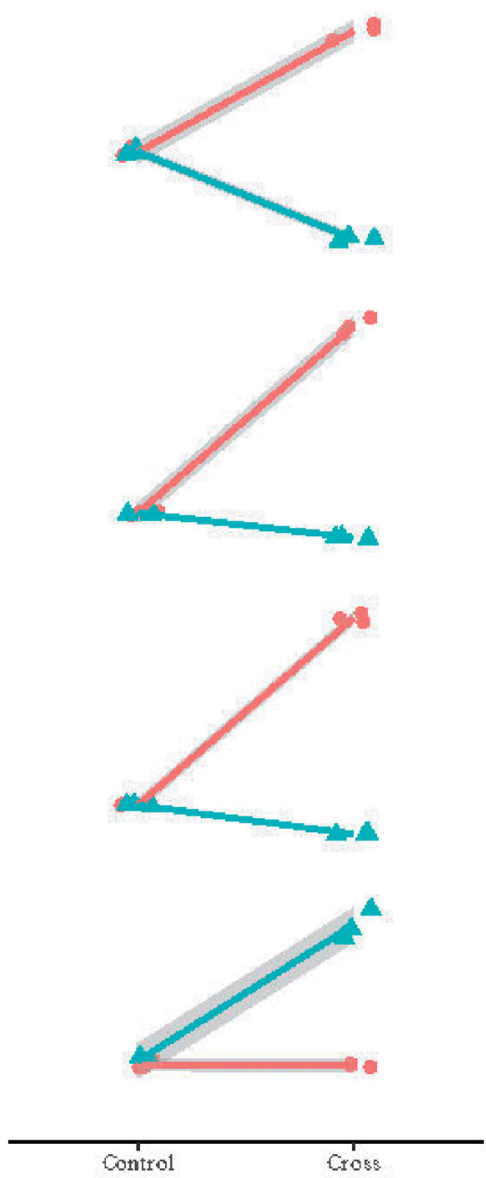

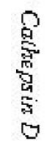

$\underset{\infty}{\infty}$

5 Days

25 Days



空

Figure 2. Representing the raw data of autophagy-related gene expression in three independent biological repeats of A 342 or Tau R406W transgenic flies and the fitted Bayesian hierarchical model lines for each group and subgroup shaded by their $95 \%$ HPD region based on 2000 posterior samplings of model parameters.

Table 1. Bayesian contrast estimation of medians (control-cross) for climbing assay in 5- and 25-day-old flies.

\begin{tabular}{clccc}
\hline Time point & Fly type & Contrast estimate (control-cross) & Lower HPD (2.5\%) & Upper HPD (97.5\%) \\
\hline \multirow{2}{*}{5 days } & A $\beta 42$ & $29.92^{\mathrm{a}}$ & 27.55 & 32.12 \\
& Tau R406W & $39.49^{\mathrm{a}}$ & 37.13 & 41.67 \\
& days & $34.83^{\mathrm{a}}$ & 32.44 & 37.04 \\
& Tau R406W & $42.53^{\mathrm{a}}$ & 40.15 & 44.74 \\
\hline
\end{tabular}

${ }^{\mathrm{a}}$ The $95 \%$ highest posterior density (HPD) intervals for each contrast are reported. Therefore, there is a significant difference between control and cross with $5 \%$ error type I $(\alpha=0.05)$ in all of the cases thus marked.

with regular eye ommatidia (blue and green colour). Interestingly, both $A \beta 42$ and Tau R406W transgenic flies demonstrate irregularities in their eyes.

\section{Quantitative real time PCR}

\section{Alteration in the expression of Hook, as a pre-autophagy gene}

As mentioned earlier, Hook is a mediator to facilitate interaction between motor proteins and their cargo as a preautophagy protein (37). In the AD brain, reduction in Hook3 expression and increase in Hook2 mRNA levels have been observed. Interestingly, when Hook3 is knocked down, there is an increase in $A \beta$ production (38). While there are three forms of Hook in humans, only one form of Hook has been reported in Drosophila. Here, we assessed the effect of $\mathrm{A} \beta 42$ and Tau R406W on the expression levels of dHook (Drosophila Hook) using transgenic flies. As can be seen in Figure 2 and from Bayesian contrast estimations in Table 2, although there was no significant change in the levels of dHook expression in 5-day-old flies expressing Tau R406W, a prominent increase was observed in the levels of this gene in 25-day-old flies. It seems that Tau R406W exerts its effect on the Hook expression at a later time point of the life cycle of this fly. Regarding A $\beta 42$-expressing flies, there was a significant decrease in the levels of Hook expression in both 5- 
(A)





Figure 3. Eye degeneration has been observed in flies expressing Tau R406W or A 342 . A and B, respectively, are analyses for eye ommatidium regularity in 5- and 25-day-old flies by ImageJ, and columns A, B, and C are GMR-GAL4/+, GMR-GAL4/Tau R406W, and GMR-GAL4/A 342 . (PP= probability parameter)

Table 2. Bayesian contrast estimation for medians of genes expressions (control-cross) in 5- and 25-day-old flies and different genes and fly types.

\begin{tabular}{|c|c|c|c|c|c|}
\hline Gene type & Time point & Fly type & Contrast estimate (control-cross) & Lower HPD (2.5\%) & Upper HPD (97.5\%) \\
\hline \multirow[t]{3}{*}{ Hook } & 5 days & $A \beta 42$ & $0.512^{\mathrm{a}}$ & 0.3964 & 0.625 \\
\hline & 25 days & $A \beta 42$ & $0.647^{\mathrm{a}}$ & 0.525 & 0.764 \\
\hline & & Tau R406W & $-1.354^{\mathrm{a}}$ & -1.475 & -1.235 \\
\hline \multirow{3}{*}{ Atg6 } & & Tau R406W & $-5.353^{\mathrm{a}}$ & -5.470 & -5.231 \\
\hline & 25 days & $A \beta 42$ & $0.569^{\mathrm{a}}$ & 0.444 & 0.681 \\
\hline & & Tau R406W & $0.829^{\mathrm{a}}$ & 0.716 & 0.951 \\
\hline & 25 days & $A \beta 42$ & $0.452^{\mathrm{a}}$ & 0.335 & 0.567 \\
\hline & & Tau R406W & $0.685^{\mathrm{a}}$ & 0.567 & 0.803 \\
\hline \multirow[t]{4}{*}{ Cathepsin D } & 5 days & $A \beta 42$ & 0.058 & -0.065 & 0.173 \\
\hline & & Tau R406W & $-1.196^{\mathrm{a}}$ & -1.309 & -1.078 \\
\hline & 25 days & $A \beta 42$ & $0.683^{a}$ & 0.564 & 0.800 \\
\hline & & Tau R406W & $0.858^{\mathrm{a}}$ & 0.743 & 0.977 \\
\hline
\end{tabular}

${ }^{a}$ The $95 \%$ highest posterior density (HPD) intervals for each contrast are reported. Therefore, there is a significant difference between control and cross with $5 \%$ error type I $(\alpha=0.05)$ in all of the cases thus marked.

and 25-day-old flies. For more details see Figure 4; and also Supplementary Table S4.

Regarding differential expression, there was about a 2.26fold increase in the Hook expression in 25-day-old flies expressing Tau R406W $(p=0.018)$. The reduction in the expression of Hook was around 0.5- and 0.59-fold for 5- and 25 -day-old A $\beta 42$-expressing flies, respectively ( $p=0.001$ and 0.002). More details are in the Supplementary data, Supplementary Figure S2 and Supplementary Table S5.

\section{Alteration in the expression of autophagy genes}

Expression of autophagy genes in fly is influenced by various factors like age (39) and oxidative stress (40). On the other hand, it has been shown that suppression or enhancement of some autophagy genes like Atg8 can increase the sensitivity to aging and oxidative stress (41). Here, the expression levels of three autophagy markers including Atg6, Atg8, and Cathepsin $D$ were assessed to track the autophagy process in
Drosophila models of AD. Transgenic flies expressing either Tau R406W or A 42 were applied as tauopathy or amyloidopathy models at two different ages, 5- and 25-day-old flies. This could provide some information on how time might affect the expression of some autophagy genes in the presence of two different types of amyloid-like aggregates.

\section{Atg6, a core protein in the nucleation stage of autophagy}

One of the main proteins for phagophore formation is Atg6. According to the Bayesian model analysis, our data showed (Figure 2 and Table 2) that Atg6 was upregulated in 5-dayold flies expressing either A $A 42$ or Tau R406W while the mRNA levels of this gene had a considerable decrease in 25day-old transgenic flies (for more details, see Figure 4; and also Supplementary Table S4). It seems that both Tau R406W and $A \beta 42$ aggregates exert the same effect on the expression levels of this gene at different time points. 

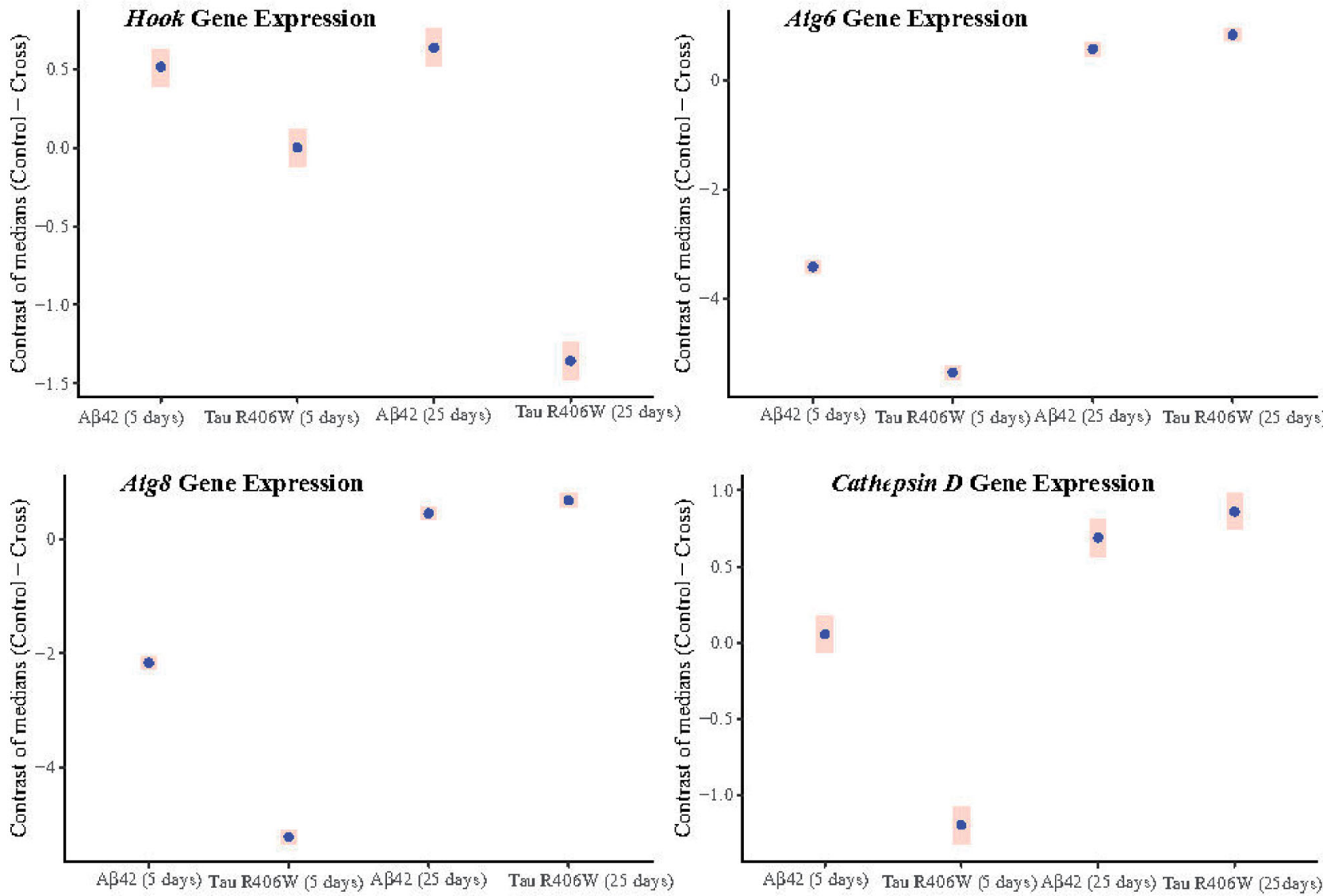

Figure 4. Bayesian estimation of median contrasts (control-cross) for gene expression in 5- and 25-day-old flies. Hook gene has different expression pattern in A 442 - or Tau R406W-expressing flies either 5 or 25 days old, while Atg6 and Atg8 mRNA levels changed with similar pattern in both transgenic lines either 5 or 25 days after eclosion.

There was a 4.45- and 6.31-fold increase in the levels of Atg6 in 5-day-old flies expressing A 442 or Tau R406W, respectively $(p \leq 0.001)$. In contrast, 25 days after eclosion these transgenic flies showed a significant decrease $(p \leq 0.001)$, around 0.58 - and 0.85 -fold, in the Atg6 expression levels compared to their counterpart controls. For more details see Supplementary Figure S2 and Supplementary Table S6.

\section{Atg8, as the main gene in the elongation phase}

Atg8 is the marker protein in the elongation phase of the autophagosome formation. This unique protein undergoes two processing steps. In the first step, Atg8 is cleaved by Atg4 and the next step is mediated by the Atg5-Atg12-Atg16 complex to get conjugated with phosphatidylethanolamine. These two steps are necessary for elongation and closure of the autophagic membrane (42). The expression of this gene showed the same pattern as the Atg6. There was a significant increase in the expression levels of Atg8 in 5-day-old transgenic flies expressing either Tau $\mathrm{R} 406 \mathrm{~W}$ or $\mathrm{A} \beta 42$, followed by a prominent decline in the levels of Atg8 mRNA expression in those flies 25 days after eclosion (for more details on the Bayesian model see Figures 2 and 4, and Table 2; and also Supplementary Table S4). Therefore, it seems that both forms of aggregates have similar impact on the Atg8 mRNA levels.
In the fold change analysis, 5-day-old A 342 or Tau R406W transgenic flies showed a 3.15- and 6.28-fold increase in the levels of Atg8 $(p \leq 0.001)$, while there was a significant decline $(p \leq 0.001)$ in the expression levels of this gene (about 0.45 - and 0.70 -fold, respectively) in 25-day-old flies expressing A $\mathrm{B} 42$ or Tau R406W (See Supplementary Figure S2 and Supplementary Table S7 for more details).

\section{Expression of the lysosome enzyme Cathepsin D, involved in the final step of autophagy}

In the central nervous system, the activity of Cathepsin D is essential to control neuronal homeostasis, cell migration, and interneuron communication. Cathepsin D-mediated proteolysis plays a significant role in neuronal survival by accomplishing the degradation of aggregated proteins that reach the lysosomes via autophagy (43). There is evidence to reveal that this protein is involved in amyloidogenic processing of the amyloid precursor protein (APP), as a critical component of $\alpha, \beta$, and $\gamma$ secretase (44). We found that the mRNA levels of Cathepsin D have different patterns in 5-day-old flies expressing Tau R406W or A $\beta 42$. According to the Bayesian model, there was no significant change in the levels of Cathepsin D mRNA in flies expressing $A \beta 42$, while Tau R406W-expressing flies showed a prominent increase in the mRNA levels of this gene at day 5 after eclosion. Interestingly, at day 25, both transgenic flies showed a 
remarkable decline in the expression levels of Cathepsin D mRNA (Figures 2 and 4, and Table 2; and also Supplementary Table S4).

The fold change analysis showed that there was a 2.24fold increase $(p=0.007)$ in the levels of Cathepsin D expression in 5-day-old flies expressing Tau R406W. In 25-day-old $\mathrm{A} \beta 42$ or Tau R406W-expressing flies, there was a 0.74 - and 0.85 -fold decrease, respectively $(p \leq 0.001)$. More details are included in Supplementary Figure S2 and Supplementary Table S8.

\section{Discussion}

According to the amyloid cascade, proposed by Hardy and Higgins in 1992, amyloid is the main culprit in AD pathology (44). Other events in $A D$, including tau hyperphosphorylation and subsequent neuronal death, were supposed to be downstream events of the amyloid pathway (45-47). Therefore, most treatments for $A D$ have been based on the removal of $A \beta$ (reviewed in 46). Recently, there is increasing evidence to support the idea that pathological tau can exert its own toxic effect independently (48-50). On the other hand, there are some studies showing that aggregations of $A \beta$ (senile plaque, SP) and tau (neurofibrillary tangles, NFT) are, in fact, a defensive mechanism against $A \beta$ monomers and the soluble pathological tau $(3,51)$. However, these aggregates, in turn, block axonal/dendritic transport (5) and can lead to mitochondrial damage and an increase in the levels of $\operatorname{ROS}(6,7)$.

In order to shed light on the mechanism underlying the relation between protein aggregates and autophagy dysfunction in $A D$, here, we investigated the differential expression of autophagy genes in A $\beta 42$-expressing flies as an amyloidopathy model and flies expressing Tau R406W as a tauopathy model (24). We used Tau R406W transgenic flies as they have been reported to exhibit AD-like phenotypes (52). On the other hand, previous studies have shown that tau wildtype transgenic flies have no aggregate formation (53). Therefore, to clarify the effect of the aggregates of this protein on autophagy genes, this model was more suitable.

Our climbing assay and fly eye analysis showed that $A \beta 42$ and Tau R406W can cause defects in climbing ability and eye regularity in transgenic flies. These data also showed that tau can exert its toxicity independent of $A \beta 42$.

Neurons are highly dependent on the autophagy to remove protein aggregates such as SP and NFT (8). Defects in this pathway clearly lead to neurodegenerative disorders such as AD. There is strong evidence to confirm the association of autophagy dysregulation with AD (54). Herrmann and colleagues showed that Hook3 was co-localized with tau aggregate and retained in those aggregates (38). Surprisingly, in our current research, there was a remarkable increase in the levels of $d$ Hook expression in 25-day-old flies expressing Tau R406W. It is possible that Hook is the adaptor for tau aggregates in the autophagy pathway and that is why the expression of Hook is increased in Tau R406W-expressing flies. However, the expression of this gene is downregulated in A $\beta 42$-expressing flies. This may suggest that either the transportation of $A \beta$ aggregates is mainly managed by another adaptor or A 342 can affect autophagy through Hook downregulation. In summary, it seems that although both Tau R406W and A 342 alter the expression of Hook, their effects occur via different mechanisms.

Several studies have shown that Beclin1 (ATG6), a main protein to initiate autophagy, is downregulated in $A D$, while some other evidence argues that autophagy genes are increased during AD $(20,55,56)$. Despite all the controversies, it is clear that autophagy is deregulated in this disease.

Atg6 is downregulated in early and late stages of AD (55). However, it has been shown that ATG8 expression is increased in $A D$ patients $(56,57)$. According to our results, while Atg6 and Atg8 showed upregulation in their mRNA levels in 5-day-old flies expressing either Tau R406W or A $\beta 42$, a remarkable downregulation was observed for both genes at day 25 after eclosion. It appears that in earlier stages ( 5 day after eclosion) autophagy genes are increased in order to clear the aggregates. However, ultimately autophagy is decreased at later time points, probably due to an increase in the ROS production $(6,7)$ or other mechanisms.

Association of autophagosome with lysosome is the last step of autophagy. Finally, its internal components are degraded by lysosome hydrolases like Cathepsin D (17). Cathepsin $D$ is the only proteolytic enzyme the expression of which, in different tissues, is regulated in response to growth factors, cytokines, and vitamins (58). Cathepsin D-mediated proteolysis is essential to neurons because it degrades unfolded/oxidized protein aggregates that continuously reach the lysosomes via autophagy or endocytosis (43). Many proteins produced in neurons are physiologic substrates of Cathepsin D and will be abnormally accumulated if they are not efficiently degraded (e.g. APP, $\alpha$-synuclein, and huntingtin). Therefore, dysfunction of Cathepsin D in the lysosomal system is closely related to the mechanism underpinning neurodegeneration (43).

In 2006, Urbanelli et al. demonstrated that there was a decrease in the Cathepsin D mRNA and protein levels in AD (59). However, in 1995, Cataldo et al. demonstrated intense Cathepsin D immunoreactivities and lowered Cathepsin D mRNA levels in degenerating neurons (60). The upregulation of Cathepsin $D$ synthesis and accumulation of hydrolaseladen lysosomes indicated an early activation of the endosomal-lysosomal system in vulnerable neuronal populations, possibly reflecting early regenerative or repair processes (60). Urbanelli and colleagues provided evidence of altered balance of the Cathepsin $D$ expression in skin fibroblasts from patients with sporadic or familial forms of AD (59). In particular, they showed that the expression of this gene is downregulated at both the transcriptional and translational levels and its processing is altered in AD fibroblasts (59). High levels of the constitutively active form of Ras in normal or AD fibroblasts induce Cathepsin D downregulation. Furthermore, the p38 MAPK signalling pathway also appears to downregulate the Cathepsin $D$ levels. Urbanelli et al. proposed that the impairment of lysosomes in AD can be one of the main factors for the progression of the disease (59). 
Finally, in 2018 Chai et al. investigated the Cathepsin D immunoreactivities in the temporal and parietal cortices of well characterized $A D$ brains. Their results showed an increase in the Cathepsin $D$ immunoreactivities in $A D$ tissues and its correlation with neuropathological NFT scores, and phosphorylated pSer396 tau burden (17).

Our data showed that there was a significant increase in the mRNA levels of Cathepsin D in Tau R406W-expressing flies just 5 days after eclosion. This increase could be due to regenerative or repair processes occurring at earlier stages of the disease (60). However, in 25-day-old transgenic flies, this gene showed significant reduction. Following the increase in Tau R406W and A 342 aggregations at later time points (25day-old flies), probably due to an increase in ROS production, Cathepsin D is downregulated (43). Moreover, cystatin C, as an inhibitor of cathepsins (cysteine protease), is upregulated by ROS and has been shown to be co-localized with the A $\beta 42$ peptide $(61,62)$.

\section{Conclusion}

In summary, our results suggest that both $A \beta 42$ and Tau R406W can affect the autophagy pathway through gene expression dysregulation. Interestingly, they showed a similar effect on the genes involved in the nucleation and elongation steps of autophagy. However, Tau R406W and A $\beta 42$ exert different effects on the expression of the pre-autophagy gene, Hook, and a gene involved in the last step of autophagy, Cathepsin D. We conclude that although both Tau R406W and $A \beta 42$ can alter the process of autophagy during $A D$, it seems that they act independently, through different mechanisms. Therefore, $A D$ treatment involving the removal of merely $A \beta 42$, without considering pathological tau, will not be sufficiently effective in ameliorating AD symptoms.

\section{Acknowledgements}

We would like to show our gratitude and appreciation to Dr. M. Haddadi for providing the desired Drosophila stock and sharing his pearls of wisdom with us during the course of working with the flies. We also express our sincere thanks to $\operatorname{Dr} M$. Ebrahimi for assistance with FLEYE plugin.

\section{Disclosure statement}

No potential conflict of interest was reported by the author(s).

\section{Funding}

This research was financially supported by Shiraz University Research Council. Seyed Morteza Najibi was supported by a Swedish Research Council grant (2016-06947) and a grant from eSSENCE@LU.

\section{Notes on contributors}

Mehrnaz Haghi, PhD student of Molecular and Cellular Biology, Department of Biology, Shiraz University, Shiraz, Iran.
Raheleh Masoudi, PhD of Molecular Genetics, Assistant professor in Department of Biology, Shiraz University, Shiraz, Iran.

Seyed Morteza Najibi, PhD of Statistics, Researcher at Center for Molecular Protein Science, Lund University, Lund, 22362, Sweden.

\section{References}

1. Hayashi SI, Sato N, Yamamoto A, Ikegame $\mathrm{Y}$, Nakashima S, Ogihara $\mathrm{T}$, et al. Alzheimer disease-associated peptide, amyloid beta40, inhibits vascular regeneration with induction of endothelial autophagy. Arterioscler Thromb Vasc Biol. 2009;29:1909-15.

2. Caccamo A, Maldonado MA, Majumder S, Medina DX, Holbein W, Magrí A, et al. Naturally secreted amyloid-beta increases mammalian target of rapamycin (mTOR) activity via a PRAS40-mediated mechanism. J Biol Chem. 2011;286:8924-32.

3. Esparza TJ, Gangolli M, Cairns NJ, Brody DL. Soluble amyloid-beta buffering by plaques in Alzheimer disease dementia versus highpathology controls. PLoS One. 2018;13:e0200251.

4. Götz J, Ittner LM, Fändrich $M$, Schonrock N. Is tau aggregation toxic or protective: a sensible question in the absence of sensitive methods? J Alzheimers Dis. 2008;14:423-9.

5. Bendiske J, Bahr BA. Lysosomal activation is a compensatory response against protein accumulation and associated synaptopathogenesis-an approach for slowing Alzheimer disease? J Neuropathol Exp Neurol. 2003;62:451-63.

6. Golpich M, Amini E, Mohamed Z, Azman Ali R, Mohamed Ibrahim $\mathrm{N}$, Ahmadiani A. Mitochondrial dysfunction and biogenesis in neurodegenerative diseases: pathogenesis and treatment. CNS Neurosci Ther. 2017;23:5-22.

7. Schulz KL, Eckert A, Rhein V, Mai S, Haase W, Reichert AS, et al. A new link to mitochondrial impairment in tauopathies. Mol Neurobiol. 2012;46:205-16.

8. Uddin M, Stachowiak A, Mamun AA, Tzvetkov NT, Takeda S, Atanasov AG, et al. Autophagy and Alzheimer's disease: from molecular mechanisms to therapeutic implications. Front Aging Neurosci 2018;10:4.

9. Chin LS, Olzmann JA, Li L. Aggresome formation and neurodegenerative diseases: therapeutic implications. Curr Med Chem. 2008; 15:47-60.

10. Olenick MA, Tokito M, Boczkowska M, Dominguez R, Holzbaur EL. Hook adaptors induce unidirectional processive motility by enhancing the dynein-dynactin interaction. J Biol Chem. 2016;291: 18239-51.

11. Hurley $\mathrm{JH}$, Young LN. Mechanisms of autophagy initiation. Annu Rev Biochem. 2017;86:225-44.

12. Fitzwalter $\mathrm{BE}$, Thorburn A. Recent insights into cell death and autophagy. Febs J. 2015;282:4279-88.

13. Shravage BV, Hill JH, Powers CM, Wu L, Baehrecke EH. Atg6 is required for multiple vesicle trafficking pathways and hematopoiesis in Drosophila. Development. 2013;140:1321-9.

14. Reggiori F, Ungermann C. Autophagosome maturation and fusion. J Mol Biol. 2017;429:486-96.

15. Badadani M. Autophagy mechanism, regulation, functions, and disorders. ISRN Cell Biol. 2012;2012:1-11.

16. Barth S, Glick D, Macleod KF. Autophagy: assays and artifacts. J Pathol. 2010;221:117-24.

17. Chai YL, Chong JR, Weng J, Howlett D, Halsey A, Lee JH, et al. Lysosomal cathepsin $\mathrm{D}$ is upregulated in Alzheimer's disease neocortex and may be a marker for neurofibrillary degeneration. Brain Pathol. 2019;29:63-74.

18. Kuchitsu Y, Fukuda M. Revisiting Rab7 functions in mammalian autophagy: Rab7 knockout studies. Cells 2018;7:215.

19. Fujita N, Huang W, Lin TH, Groulx JF, Jean S, Nguyen J, et al. Genetic screen in Drosophila muscle identifies autophagy-mediated T-tubule remodeling and a Rab2 role in autophagy. Elife. 2017;6:e23367.

20. Lipinski MM, Zheng B, Lu T, Yan Z, Py BF, Ng A, et al. Genomewide analysis reveals mechanisms modulating autophagy in 
normal brain aging and in Alzheimer's disease. Proc Natl Acad Sci USA. 2010;107:14164-9.

21. Burgoyne JR. Oxidative stress impairs autophagy through oxidation of ATG3 and ATG7. Autophagy 2018;14:1092-3.

22. Kaur U, Banerjee P, Bir A, Sinha M, Biswas A, Chakrabarti S. Reactive oxygen species, redox signaling and neuroinflammation in Alzheimer's disease: the NF-KB connection. Curr Top Med Chem. 2015;15:446-57.

23. Sarkar S, Korolchuk VI, Renna M, Imarisio S, Fleming A, Williams A et al. Complex inhibitory effects of nitric oxide on autophagy. Mol Cell. 2011;43:19-32.

24. Wittmann CW, Wszolek MF, Shulman JM, Salvaterra PM, Lewis J, Hutton $M$, et al. Tauopathy in Drosophila: neurodegeneration without neurofibrillary tangles. Science. 2001;293:711-4.

25. Tare M, Modi RM, Nainaparampil JJ, Puli OR, Bedi S, FernandezFunez $\mathrm{P}$, et al. Activation of JNK signaling mediates amyloid-ssdependent cell death. PLOS One. 2011;6:e24361.

26. Chouhan AK, Guo C, Hsieh YC, Ye H, Senturk M, Zuo Z, et al. Uncoupling neuronal death and dysfunction in Drosophila models of neurodegenerative disease. Acta Neuropathol Commun. 2016;4: 62.

27. Finelli A, Kelkar A, Song HJ, Yang $H$, Konsolaki M. A model for studying Alzheimer's Abeta42-induced toxicity in Drosophila melanogaster. Mol Cell Neurosci. 2004;26:365-75.

28. Diez-Hermano S, Valero J, Rueda C, Ganfornina MD, Sanchez D. An automated image analysis method to measure regularity in biological patterns: a case study in a Drosophila neurodegenerative model. Mol Neurodegener. 2015;10:9.

29. Madabattula ST, Strautman JC, Bysice AM, O'Sullivan JA Androschuk A, Rosenfelt C. Quantitative analysis of climbing defects in a Drosophila model of neurodegenerative disorders. J Vis Exp 2015;100:e52741.

30. Ali YO, Escala W, Ruan K, Zhai RG. Assaying locomotor, learning, and memory deficits in Drosophila models of neurodegeneration. $J$ Vis Exp 2011;49:2504.

31. Koopmans M, Monroe SS, Coffield LM, Zaki SR. Optimization of extraction and PCR amplification of RNA extracts from paraffinembedded tissue in different fixatives. J Virol Methods. 1993;43: 189-204

32. Fukatsu T. Acetone preservation: a practical technique for molecular analysis. Mol Ecol. 1999;8:1935-45.

33. Livak KJ, Schmittgen TD. Analysis of relative gene expression data using real-time quantitative $P C R$ and the 2(-delta delta $C(T)$ ) method. Methods. 2001;25:402-8.

34. Austin PC, Brunner LJ, Hux JE. Bayeswatch: an overview of Bayesian statistics. J Eval Clin Pract. 2002;8:277-86.

35. Button KS, loannidis JP, Mokrysz C, Nosek BA, Flint J, Robinson ES, et al. Power failure: why small sample size undermines the reliability of neuroscience. Nat Rev Neurosci. 2013;14:365-76.

36. R Core Team. R: A language and environment for statistical computing. Vienna, Austria: R Foundation for Statistical Computing; 2020. Available at: http://www.R-project.org/.

37. Szebenyi G, Hall B, Yu R, Hashim Al, Krämer H. Hook2 localizes to the centrosome, binds directly to centriolin/CEP110 and contributes to centrosomal function. Traffic. 2007;8:32-46.

38. Herrmann L, Wiegmann C, Arsalan-Werner A, Hilbrich I, Jäger C, Flach $\mathrm{K}$, et al. Hook proteins: association with Alzheimer pathology and regulatory role of hook3 in amyloid beta generation. PLOS One. 2015;10:e0119423.

39. Rubinsztein DC, Shpilka T, Elazar Z. Mechanisms of autophagosome biogenesis. Curr Biol. 2012;22:R29-R34.

40. Simonsen A, Cumming RC, Brech A, Isakson P, Schubert DR, Finley $\mathrm{KD}$. Promoting basal levels of autophagy in the nervous system enhances longevity and oxidant resistance in adult Drosophila. Autophagy 2008;4:176-84.
41. Wu H, Wang MC, Bohmann D. JNK protects Drosophila from oxidative stress by trancriptionally activating autophagy. Mech Dev. 2009;126:624-37.

42. Shpilka T, Weidberg H, Pietrokovski S, Elazar Z. Atg8: an autophagy-related ubiquitin-like protein family. Genome Biol. 2011;12: 226.

43. Di Domenico F, Tramutola A, Perluigi M. Cathepsin D as a therapeutic target in Alzheimer's disease. Expert Opin Ther Targets. 2016;20:1393-5.

44. Straface E, Matarrese P, Gambardella L, Vona R, Sgadari A, Silveri $M C$, et al. Oxidative imbalance and cathepsin $D$ changes as peripheral blood biomarkers of Alzheimer disease: a pilot study. FEBS Lett. 2005;579:2759-66.

45. Hardy JA, Higgins GA. Alzheimer's disease: the amyloid cascade hypothesis. Science. 1992;256:184-5.

46. Lewis J, Dickson DW, Lin WL, Chisholm L, Corral A, Jones G, et al Enhanced neurofibrillary degeneration in transgenic mice expressing mutant tau and APP. Science. 2001;293:1487-91.

47. Rajmohan R, Reddy PH. Amyloid-beta and phosphorylated tau accumulations cause abnormalities at synapses of Alzheimer's disease neurons. J Alzheimers Dis. 2017;57:975-99.

48. Kametani F, Hasegawa M. Reconsideration of amyloid hypothesis and tau hypothesis in Alzheimer's Disease. Front Neurosci. 2018, 12:25.

49. Belrose JC, Masoudi R, Michalski B, Fahnestock M. Increased pronerve growth factor and decreased brain-derived neurotrophic factor in non-Alzheimer's disease tauopathies. Neurobiol Aging. 2014;35:926-33.

50. Harrison JR, Owen MJ. Alzheimer's disease: the amyloid hypothesis on trial. Br J Psychiatry 2016;208:1-3.

51. Bretteville A, Planel E. Tau aggregates: toxic, inert, or protective species? J Alzheimers Dis. 2008;14:431-6.

52. Nakamura $M$, Shiozawa $S$, Tsuboi $D$, Amano $M$, Watanabe $H$, Maeda $\mathrm{S}$, et al. Pathological progression induced by the frontotemporal dementia-associated R406W tau mutation in patientderived iPSCs. Stem Cell Reports. 2019;13:684-99.

53. Passarella D, Goedert M. Beta-sheet assembly of tau and neurodegeneration in Drosophila melanogaster. Neurobiol Aging. 2018;72: 98-105.

54. Funderburk SF, Marcellino BK, Yue Z. Cell "'self-eating' (autophagy) mechanism in Alzheimer's disease". Mt Sinai J Med. 2010;77:59-68.

55. Lee JA, Gao FB. Regulation of Abeta pathology by beclin 1: a protective role for autophagy? J Clin Invest. 2008;118:2015-8.

56. Ihara $Y$, Morishima-Kawashima M, Nixon R. The ubiquitin-proteasome system and the autophagic-lysosomal system in Alzheimer disease. Cold Spring Harb Perspect Med. 2012;2:a006361.

57. Rajaguru P, Vaiphei K, Saikia B, Kochhar R. Increased accumulation of dendritic cells in celiac disease associates with increased expression of autophagy protein LC3. Indian J Pathol Microbiol. 2013;56:342.

58. Vidoni $C$, Follo $C$, Savino $M$, Melone MAB, Isidoro $C$. The role of cathepsin $D$ in the pathogenesis of human neurodegenerative disorders. Med Res Rev. 2016;36:845-70.

59. Urbanelli L, Emiliani C, Massini C, Persichetti E, Orlacchio A, Pelicci $\mathrm{G}$, et al. Cathepsin D expression is decreased in Alzheimer's disease fibroblasts. Neurobiol Aging. 2008;29:12-22.

60. Cataldo AM, Barnett JL, Berman SA, Li J, Quarless S, Bursztajn S, et al. Gene expression and cellular content of cathepsin D in Alzheimer's disease brain: evidence for early up-regulation of the endosomal-lysosomal system. Neuron 1995;14:671-80.

61. McGrath LT, McGleenon BM, Brennan S, McColl D, Mcllroy S, Passmore AP. Increased oxidative stress in Alzheimer's disease as assessed with 4-hydroxynonenal but not malondialdehyde. QJM. 2001;94:485-90.

62. Christen Y. Oxidative stress and Alzheimer disease. Am J Clin Nutr. 2000;71:621S-9S. 